\title{
Antidepressant effect of venlafaxine in chronic unpredictable stress: Evidence of the involvement of key enzymes responsible for monoamine neurotransmitter synthesis and metabolism
}

\author{
DAN LIU ${ }^{1}$, XIAO-YA HU ${ }^{2}$, HAI-JIAN XIA ${ }^{3}$, LI-JIA WANG ${ }^{2}$, \\ PING SHI $^{1}$, XIANG-PAN CHEN ${ }^{1}$ and QI-XIN ZHOU ${ }^{4}$ \\ ${ }^{1}$ Department of Pharmacy, Chongqing General Hospital, Chongqing 400013; \\ Departments of ${ }^{2}$ Pharmacy and ${ }^{3}$ Neurosurgery, First Affiliated Hospital of Chongqing Medical University; \\ ${ }^{4}$ Key Laboratory of Biochemistry and Molecular Pharmacology, Department of Pharmacology, \\ Chongqing Medical University, Chongqing 400016, P.R. China
}

Received September 26, 2018; Accepted June 12, 2019

DOI: $10.3892 / \mathrm{mmr} .2019 .10489$

\begin{abstract}
A number of studies have linked abnormalities in the function of the serotonergic and noradrenergic systems to the pathophysiology of depression. It has been reported that selective serotonin reuptake inhibitors promote the expression of tryptophan hydroxylase (TPH), which is involved in the synthesis of serotonin. However, limited evidence of TPH alteration has been found in selective serotonin and noradrenaline reuptake inhibitors (SNRIs), and more key enzymes need to be investigated. The aim of the present study was to determine whether venlafaxine (VLX; a classical SNRI) regulates TPH and other key enzymes responsible for the synthesis and metabolism of monoaminergic transmitters in rats with chronic unpredictable stress (CUS). The present results suggested that CUS-exposed rats exhibited decreased locomotor activity in the open-field test and increased immobility time in the forced swim test, as compared with the controls. Pretreatment with VLX (20 mg/kg) significantly increased locomotor activity and reduced immobility time in the CUS-exposed rats. In addition, VLX $(20 \mathrm{mg} / \mathrm{kg})$ treatment prevented the CUS-induced reduction in tyrosine hydroxylase and TPH expression in the cortex and hippocampus. Furthermore, VLX alleviated the CUS-induced oxidative stress in the serum, cortex and hippocampus. However, VLX administration did not have an
\end{abstract}

Correspondence to: Professor Qi-Xin Zhou, Key Laboratory of Biochemistry and Molecular Pharmacology, Department of Pharmacology, Chongqing Medical University, 1 Yixueyuan Road, Yuzhong, Chongqing 400016, P.R. China

E-mail: cqzhouqixin@163.com

Key words: tyrosine hydroxylase, tryptophan hydroxylase, indoleamine-2,3-dioxygenase, monoamine oxidase A, chronic unpredictable stress effect on indoleamine-2,3-dioxygenase overexpression in the hippocampus. It was therefore concluded that the regulation of abnormalities in the synthesis and metabolism of monoaminergic transmitters may be associated with the antidepressant effects of VLX, suggesting that multimodal pharmacological treatments can efficiently treat depression.

\section{Introduction}

Abnormalities in monoamine synthesis and transmission have, for a long time, been implicated in the pathogenesis of depressive disorder (1). Tyrosine hydroxylase (TH) and tryptophan hydroxylase (TPH) are rate-limiting enzymes in catecholamine and serotonin biosyntheses, respectively and their level is closely linked to the etiology and development of depression. A previous clinical study showed that the $\mathrm{TH}$ immunoreactivity was lower $(\sim 30 \%)$ in the locus coeruleus of suicide victims (2). It was found that chronic mild stress resulted in depressive behavior in rats with decreased TH mRNA expression in the locus coeruleus in both sexes (3). In the brain of male C57BL/6 mice, it was found that chronic fluoxetine treatment increased locus coeruleus TH (4). Meanwhile, it was evidenced that TPH activity was significantly decreased in the hippocampus of mice following the induction of chronic stress over 20 days (5). In TPH-2 knockout and TPH1/TPH2 double knockout mice, central serotonin (5-HT) levels were markedly reduced with associated depressive behaviors (6). Previous studies have reported that fluoxetine and sertraline contribute to an increase in TPH expression (7-9). However, further studies focusing on the association between the serotonergic/noradrenergic systems and the mechanisms of action of antidepressants are still required.

It was recently suggested that the indoleamine-2,3dioxygenase (IDO) pathway of tryptophan metabolism may be involved in the onset of depression $(10,11)$. IDO is an extra-hepatic rate-limiting enzyme, which catalyzes the metabolism of tryptophan along the kynurenine pathways (12). 
The activation of IDO leads to tryptophan depletion and the accumulation of kynurenine pathway-induced neurotoxic metabolites, including kynurenic acid, quinolinic acid and 3-hydroxy kynurenine; both of these changes are considered to be associated with the progression of depression $(13,14)$. In lipopolysaccharide injection-induced depressive mice, upregulation of IDO expression may be observed in the brainstem, as well as an increased kynurenine/tryptophan ratio in the serum (15). On the other hand, neurotoxic metabolites of IDO extensively disturb neurotransmission by releasing oxidative stress mediators. In fact, the brain is particularly vulnerable to oxidative/nitrosative stress in neurodegenerative disorders. Stefanescu et al (16), reported increased malondialdehyde (MDA) content with decreased superoxide dismutase (SOD) activity in the serum of depressed patients (particularly in patients with recurrent symptoms), which could be reversed by treatment with venlafaxine (VLX) and citalopram.

Type A monoamine oxidase (MAO-A) is an enzyme associated with monoamine transmitter metabolism, and plays a vital role in the onset, development and treatment of depression (17). The MAO-A level in the brain is determined prior to birth, and MAO-A and its major substrate, 5-HT, regulate the development of the neuronal architecture (18). A clinical study of 17 depressed individuals concluded that MAO-A levels in the brain were elevated during untreated major depression and that this was the primary monoamine-lowering process (19). Following exposure to chronic social defeat stress, a significant increase was observed in rat serum corticosterone (glucocorticoids) levels, which was correlated with the upregulation of MAO-A (20). In fact, the reversible inhibitor of MAO-A moclobemide has shown an antidepressant effect in the treatment of a wide spectrum of depressive disorders (21).

Selective serotonin and noradrenaline reuptake inhibitors (SNRIs) are a major class of antidepressants, and their therapeutic effect is generally attributed to an increase in the availability of monoamines in the synapses between neurons (22). The efficiency of the drugs takes up to 4-6 weeks to manifest, and the understanding of this mechanism remains unclear. Whether the drugs regulate the key enzymes associated with the synthesis and metabolism of monoamine neurotransmitters needs to be further evaluated. In the present study, the expression of TH, TPH, IDO and MAO-A, as well as the oxidative stress levels in response to treatment with VLX (a classical SNRI), were measured in a rat model of CUS-induced depression.

\section{Materials and methods}

Animals. A total of 80 6-week-old male Sprague-Dawley rats (Animal Experimental Center, Chongqing Medical University) weighing 180-200 $\mathrm{g}$ were housed in groups of 4 rats/cage. Before the experiment, rats were kept at $25 \pm 2{ }^{\circ} \mathrm{C}$ and a relative humidity of $55 \pm 5 \%$ in a quiet, airy and clean environment (12-h light/dark cycle) with ad libitum feeding and drinking. The animals were allowed to habituate to the room for 7 days and received health checks daily. Rats were anesthetized with $10 \%$ chloral hydrate $[30 \mathrm{mg} / 0.1 \mathrm{~kg}$; intraperitoneal (i.p.)] before being sacrificed. All experimental procedures were approved by the Ethics Committee of Chongqing Medical University.
Experimental design. A total of 80 screened rats were randomly divided into four groups $(n=20)$ : The control group; chronic unpredictable stress (CUS) group; VLX group; and VLX+CUS group. VLX (Wuhan Shengtianyu Technology Co. Ltd.) was dissolved in $0.5 \%$ sodium carboxymethyl cellulose (CMC-Na) solution to obtain a concentration of $2 \mathrm{mg} / \mathrm{ml}$. Animals in the VLX and VLX+CUS groups were treated with VLX ( $20 \mathrm{mg} / \mathrm{kg}$-day) by gavage once a day for 28 days. Control and CUS group rats were treated with same volume of CMC-Na.

CUS paradigm. CUS and VLX+CUS group rats were exposed to various stressors for 28 days, and the matched control rats did not receive any stressors. The stressors were little-modified from a method described previously (23), including 1-min nip-tail, 5-min forced swimming in ice water, 24-h cage tilting at $45^{\circ}, 24-\mathrm{h}$ wet bedding, 24-h food and water deprivation, 5-min thermal environment at $47^{\circ} \mathrm{C}, 3$-min electric shock at $45 \mathrm{~V}, 2$-h loud noise, overnight illumination, and alterations of the light-dark cycle (2-h light/dark cycle). On average, rats were individually exposed to the stressors once a day. The same stressors were never performed in succession within 4 days.

Open-field test (OFT). The locomotive activity was measured by OFT following a previously published method (24), in quiet and semi-dark conditions. A self-made wooden box $(100 \mathrm{~cm}$ $\mathrm{x} 100 \mathrm{~cm} \mathrm{x} 40 \mathrm{~cm} ; 4 \mathrm{~cm} \mathrm{x} 4 \mathrm{~cm}$ square grids in the bottom) with a black inner surface was used in this experiment. Each rat was carefully placed in the center of the bottom of the box sequentially and allowed to freely explore for $5 \mathrm{~min}$. Ambulation and rearing scores were collected by two experienced observers. The box was cleaned thoroughly between tests.

Forced swim test (FST). The FST was conducted according a reported method (25) by placing rats individually into a vertical transparent acrylic cylinder $(60 \mathrm{~cm}$ height x $30 \mathrm{~cm}$ diameter). The cylinder was filled with water at a depth of $35 \mathrm{~cm}\left(\right.$ at $23 \pm 2^{\circ} \mathrm{C}$ ) which kept the rat upright and unable to touch the bottom nor jump out of the cylinder. Each rat was forced to swim for $6 \mathrm{~min}$, and the immobile time during the first 3, last 3 and total 6 min was recorded by an observer blinded to the treatments. Immobility was defined as a state of floating (>2 sec, no climbing or swimming) with necessary stroke to keep the head above water.

Western blot analysis. On the 29th day, the animals were sacrificed by rapid decapitation and their heads were immediately snap frozen in liquid nitrogen for few seconds. In each rat, the cortex and hippocampi were rapidly dissected on an ice-cold surface, and frozen in liquid nitrogen before protein extraction. Protein samples were extracted using a Membrane and Cytosol Protein Extraction kit (cat. no. P0033; Beyotime Institute of Biotechnology) following homogenization. A bicinchoninic acid kit (cat. no. P0011; Beyotime Institute of Biotechnology) was used to measure the protein concentration. Protein samples were boiled in SDS-PAGE buffer for $5 \mathrm{~min}$. A mass of $50 \mu \mathrm{g} /$ lane total protein was separated by $10 \%$ SDS-PAGE and transferred to polyvinylidene fluoride membranes. The membrane was blocked with $5 \%$ milk in TBST (TBS with $0.05 \%$ Tween-20) for $1 \mathrm{~h}$ at room temperature. Following 

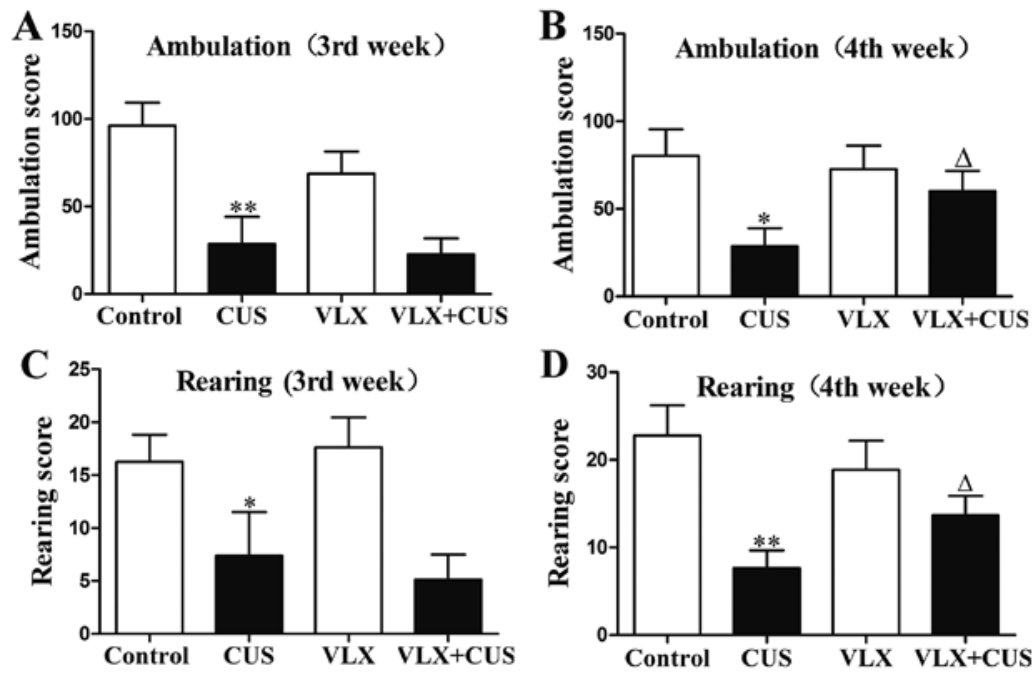

Figure 1. Effect of CUS on the scores of the rats in the open-field test, and the effect of intervention with VLX. The ambulation scores of the rats at the (A) 3rd and (B) 4th weeks are presented. The rearing scores of the rats at the (C) 3rd and (D) 4th weeks are presented. Mean \pm SEM, $n=10$. ${ }^{*} \mathrm{P}<0.05,{ }^{* *} \mathrm{P}<0.01 \mathrm{vs}$. control; ${ }^{\Delta} \mathrm{P}<0.05$ vs. CUS. CUS, chronic unpredictable stress; VLX, venlafaxine.

A

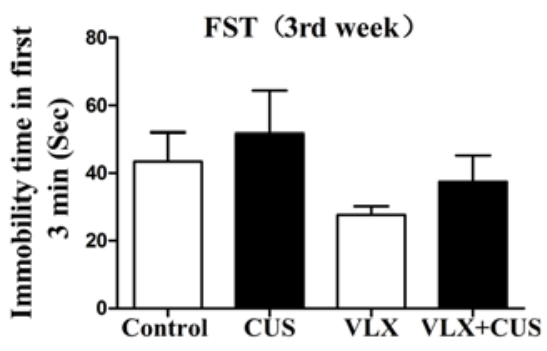

D

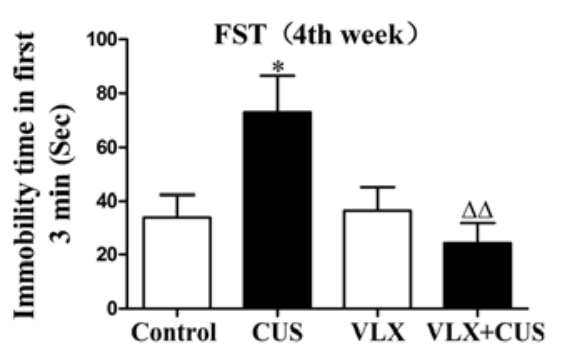

B

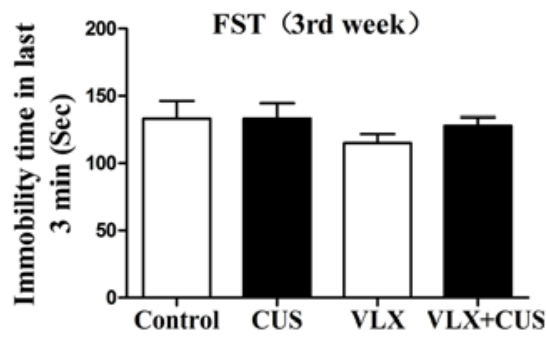

$\mathbf{E}$

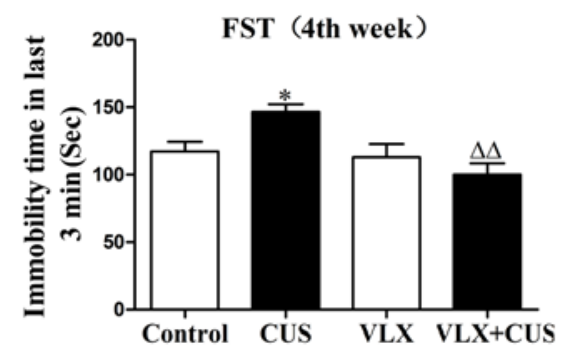

C

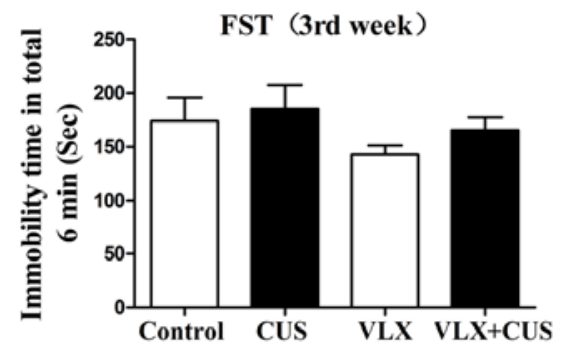

$\mathbf{F}$

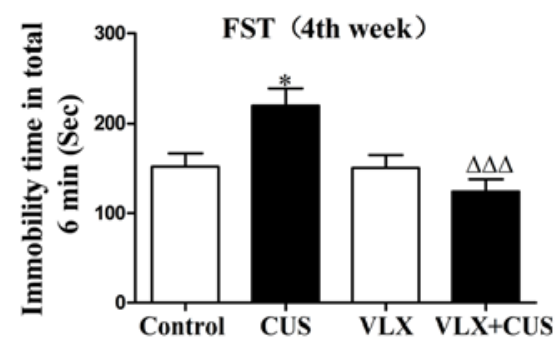

Figure 2. Effect of CUS on the immobility time of the rats in the FST, and the effect of intervention with VLX. The immobility times in (A) the first 3 min, (B) the last $3 \mathrm{~min}$ and (C) the total $6 \mathrm{~min}$ at the 3 rd week are presented. In addition, the immobility times in (D) the first 3 min, (E) the last 3 min and (F) the total $6 \mathrm{~min}$ at the 4 th week are presented. Mean $\pm \mathrm{SEM}, \mathrm{n}=10$. ${ }^{*} \mathrm{P}<0.05$ vs. control; ${ }^{\Delta \Delta} \mathrm{P}<0.01,{ }^{\Delta \Delta \Delta} \mathrm{P}<0.001$ vs. CUS. CUS, chronic unpredictable stress; VLX, venlafaxine; FST, forced swim test.

blocking, the membrane was washed with TBST, then incubated with anti-TH (cat. no. AB152) and anti-TPH (cat. no. AB1541) antibodies separately (1:1,000 dilution; EMD Millipore) overnight at $4^{\circ} \mathrm{C}$, and $\beta$-actin (cat. no. TA-09, 1:2,000 dilution, OriGene Technologies, Inc.) was established as loading control. After washing, the membranes were incubated with peroxidase-conjugated secondary antibodies (cat. no. A0208, 1:1,000 dilution, Beyotime Institute of Biotechnology; cat. no. D110174, 1:5,000 dilution, Sangon Biotech Co., Ltd.) for $1 \mathrm{~h}$ at room temperature. The membranes were developed using an enhanced chemiluminescence western blot detection system (Pierce; Thermo Fisher Scientific, Inc.). Images were acquired and analyzed using Quantity One version 4.5.2 software (Bio-Rad Laboratories, Inc.).

Reverse transcription-quantitative PCR (RT-qPCR). Tissue samples were homogenized for RNA isolation using TRIzol ${ }^{\circledR}$ (Thermo Fisher Scientific, Inc.) in combination with RNeasy Minikits (Qiagen $\mathrm{GmbH}$ ). RT was conducted using iScript cDNA synthesis kit (Bio-Rad Laboratories) for $5 \mathrm{~min}$ at $25^{\circ} \mathrm{C}$, $30 \mathrm{~min}$ at $42^{\circ} \mathrm{C}$, and $5 \mathrm{~min}$ at $85^{\circ} \mathrm{C}$. qPCR was performed using SYBR Premix Ex Taq II (Takara Biotechnology Co., Ltd.) in a Real-Time PCR detection system (Bio-Rad Laboratories). Thermocycling conditions were an initial step of $30 \mathrm{sec}$ at $95^{\circ} \mathrm{C}$ 
A

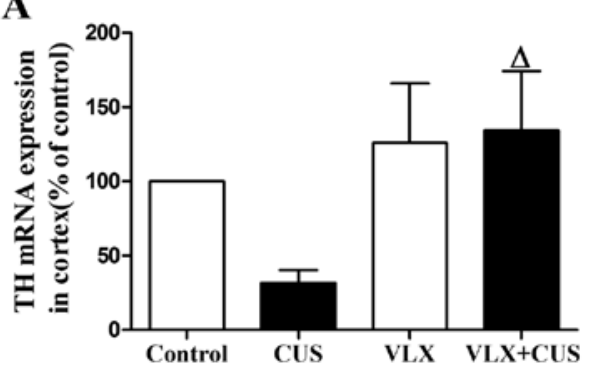

C

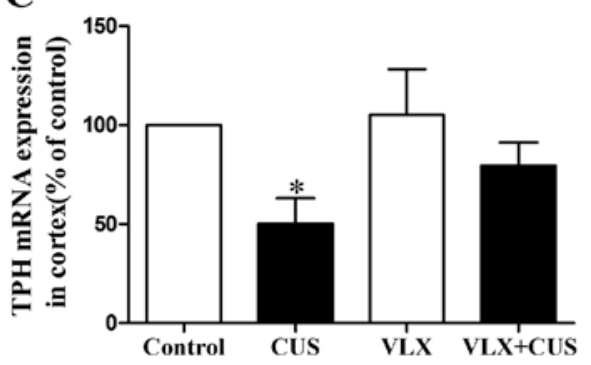

B

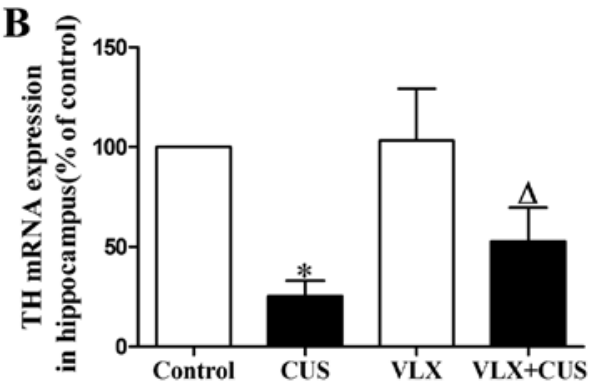

D

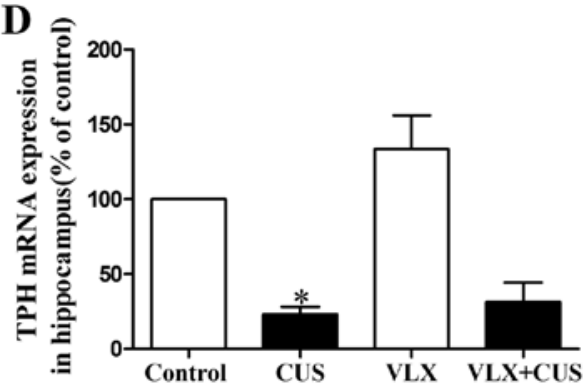

Figure 3. Effect of CUS on TH and TPH mRNA expression in the rat cortex and hippocampus, and the effect of intervention with VLX. The graphs indicate TH mRNA expression in the (A) cortex and (B) hippocampus, and TPH mRNA expression in the (C) cortex and (D) hippocampus. Mean \pm SEM, $\mathrm{n}=5$. ${ }^{*} \mathrm{P}<0.05$ vs. control; ${ }^{\triangle} \mathrm{P}<0.05$ vs. CUS. CUS, chronic unpredictable stress; VLX, venlafaxine; TH, tyrosine hydroxylase; TPH, tryptophan hydroxylase.

and 40 cycles of $5 \mathrm{sec}$ at $95^{\circ} \mathrm{C}, 30 \mathrm{sec}$ at $55^{\circ} \mathrm{C}$, and $1 \mathrm{~min}$ at $72^{\circ} \mathrm{C}$, with a final extension of $10 \mathrm{~min}$ at $72^{\circ} \mathrm{C}$. $\beta$-actin was included as a loading control in each analysis. Quantification was conducted via the $2^{-\Delta \Delta \mathrm{Cq}}$ method (26). Primer sequences were: $\beta$-actin (NM_031144.3), forward 5'-CGTAAAGACCTC TATGCCAACA-3' and reverse 5'-TAGGAGCCAGGGCAG TAATC-3'; TH (NM_012740.3), forward 5'-AGAGGACAG CATCCCACAGC-3' and reverse 5'- ATCACGGGCGGACAG TAGA-3'; TPH (NM_173839.2), forward 5'-TTTGTAGCC AACATTCCTCA-3' and reverse 5'-ACTATTGAAAGTAGA AACCACCTC-3'; IDO (NM_023973.1), forward 5'-TGATGT CCTTCTGGGAATAAA-3' and reverse 5'-AGCCTCCTT CAAGTCTTCATT-3'; and MAO-A (NM_033653.1), forward 5'-GCCAGCCAGTAGGTAGGAT-3' and reverse 5'-CTTGGA CTCGGGTTCTTCA-3'.

Determination of SOD activity and MDA content. Rat blood samples were collected after rapid decapitation and deposited at $4^{\circ} \mathrm{C}$ for $30 \mathrm{~min}$. Serum was obtained after centrifugation at $1,000 \mathrm{x} \mathrm{g}$ for $10 \mathrm{~min}$ at $4^{\circ} \mathrm{C}$ (Thermo Fisher Scientific, Inc.). The cortex and hippocampi of each rat were isolated rapidly on ice. Tissue samples were weighed and then homogenized separately with an electric tissue homogenizer (IKA Werke $\mathrm{GmbH} \& \mathrm{Co} . \mathrm{KG}$ ) in ice-cold homogenization buffer. The homogenate was centrifuged at $5,000 \mathrm{x}$ g for $10 \mathrm{~min}$ at $4^{\circ} \mathrm{C}$.The supernatant was carefully obtained for the determination of SOD activity and MDA content according to the instructions of the assay kit (Nanjing Jiancheng Bioengineering Institute).

Statistical analysis. Data from behavioral and biochemical studies are presented as the mean \pm SEM. Experiments were repeated $\geq 5$ times. All statistical analyses were conducted using GraphPad 5.0 (GraphPad Software, Inc.) via one-way ANOVA followed by the Bonferroni test for multiple comparisons. $\mathrm{P}<0.05$ was considered to indicate a statistically significant difference.

\section{Results}

Behavioral changes in the OFT and FST. In the OFT at the 3rd week, CUS rats displayed a significant decrease in both ambulation and rearing score, compared with control rats $(\mathrm{P}<0.01$, Fig. 1A; $\mathrm{P}<0.05$, Fig. 1C). Similarly, in the OFT at the 4th week, decreased ambulation and rearing scores were also observed in the CUS group compared with the control group $(\mathrm{P}<0.05$, Fig. 1B; $\mathrm{P}<0.01$, Fig. 1D). Administration of VLX prevented the decrease in ambulation and rearing score in the VLX+CUS rats at the 4 th week $(\mathrm{P}<0.05$ vs. CUS rats; Fig. $1 \mathrm{~B}$ and D).

In the FST, the immobility time of the CUS rats was significantly prolonged at the 4 th week $(\mathrm{P}<0.05$ vs. control rats; Fig. 2D-F). VLX treatment inhibited this increase in immobility time in the VLX+CUS rats at the 4th week, compared with the CUS rats $(\mathrm{P}<0.01$, Fig. $2 \mathrm{D}$ and $\mathrm{E} ; \mathrm{P}<0.001$, Fig. $2 \mathrm{~F})$.

TH and TPH mRNA expression in the cortex and hippocampus. TH mRNA expression in the hippocampus, and TPH mRNA expression in both the cortex and hippocampus were significantly decreased in the CUS rats $(\mathrm{P}<0.05$ vs. control rats; Fig. 3B-D). VLX administration clearly prevented the decrease in TH mRNA in the cortex and hippocampus in the VLX+CUS rats $(\mathrm{P}<0.05$ vs. CUS rats; Fig. $3 \mathrm{~A}$ and $\mathrm{B})$. VLX treatment tended to upregulate TPH mRNA expression in the cortex and hippocampus in VLX+CUS rats, but there was no significant difference ( $\mathrm{P}>0.05$ vs. CUS rats; Fig. $3 \mathrm{C}$ and $\mathrm{D})$.

TH and TPH protein expression in the cortex and hippocampus. Meanwhile, TH protein expression was clearly decreased in the cortex and hippocampus of CUS rats $(\mathrm{P}<0.05$ vs. control rats; Fig. $4 \mathrm{~A}$ and B). TPH protein expression in CUS rats was significantly reduced in the hippocampus $(\mathrm{P}<0.01$ vs. control rats; Fig. 4D) and tended to decrease in the 

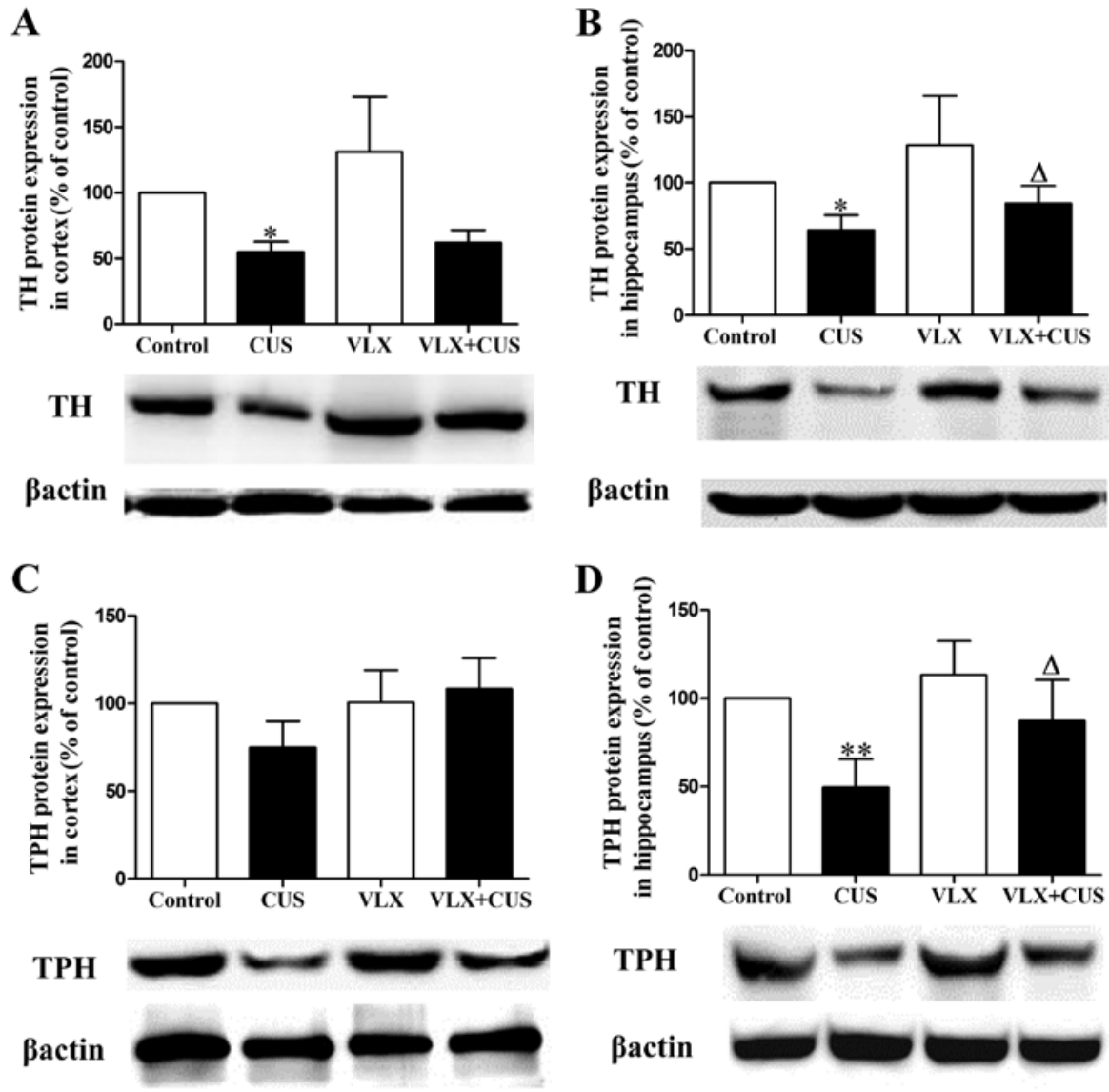

Figure 4. Effect of CUS on TH and TPH protein expression in the rat cortex and hippocampus, and the effect of intervention with VLX. TH protein expression in the (A) cortex and (B) hippocampus, and TPH protein expression in the (C) cortex and (D) hippocampus was analyzed. Mean $\pm S E M, n=5$. $P<0.05,{ }^{* * *} \mathrm{P}<0.01$ vs. control; ${ }^{\triangle} \mathrm{P}<0.05$ vs. CUS. CUS, chronic unpredictable stress; VLX, venlafaxine; $\mathrm{TH}$, tyrosine hydroxylase; TPH, tryptophan hydroxylase.

cortex ( $\mathrm{P}>0.05$ vs. control rats; Fig. 4C). VLX administration prevented the decrease in TH and TPH protein expression in the hippocampus of VLX+CUS rats $(\mathrm{P}<0.05$ vs. CUS rats; Fig. 4B and D). VLX treatment tended to increase TPH protein expression in the cortex of VLX+CUS rats, but no significant difference was observed ( $\mathrm{P}>0.05$ vs. CUS rats; Fig. $4 \mathrm{C}$ ).

mRNA expression of IDO and MAO-A. In CUS rats, IDO mRNA expression was significantly elevated and MAO-A mRNA expression tended to be upregulated compared with control rats ( $\mathrm{P}<0.01$, Fig. 5A; $\mathrm{P}>0.05$, Fig. 5B). Administration of VLX inhibited the increase in MAO-A expression but failed to have an effect on IDO overexpression in VLX+CUS rats, compared with CUS rats ( $\mathrm{P}>0.05$, Fig. 5A; $\mathrm{P}<0.05$, Fig. 5B).

Alteration in SOD activity and MDA content. Compared with control rats, the CUS rats showed significantly lower SOD activity in the serum, cortex and hippocampus $(\mathrm{P}<0.05$; Fig. 6A-C), as well as a higher MDA content in the cortex and hippocampus $(\mathrm{P}<0.05$, Fig. $6 \mathrm{E}$ and $\mathrm{F})$. There was no significant difference in MDA content of serum between CUS and control rats $(\mathrm{P}>0.05$, Fig. $6 \mathrm{D}) . \mathrm{VLX}$ intervention prevented the decrease in SOD activity in the serum and cortex in VLX+CUS rats $(\mathrm{P}<0.05$ vs. CUS rats; Fig. 6A and $\mathrm{B})$. VLX also inhibited the increase in MDA content in the cortex and hippocampus in VLX+CUS rats, compared with CUS rats $(\mathrm{P}<0.05$, Fig. $6 \mathrm{E}$; $\mathrm{P}<0.01$, Fig. $6 \mathrm{~F}$ ). However, VLX failed to decrease the MDA content of serum significantly in VLX+CUS rats $(\mathrm{P}>0.05$ vs. CUS rats, Fig. 6D).

\section{Discussion}

In the present study, it was shown that exposure to CUS for 4 weeks not only induced depression-like behaviors, but also resulted in a general downregulation of TH and TPH, as well as an upregulation of IDO and MAO-A. The results indicated that chronic stress-induced depression-like behavior may be associated with abnormalities in the synthesis and metabolism of monoaminergic transmitters. Moreover, CUS induced increased MDA content and decreased SOD activity, suggesting neuronal damage induced by oxidative stress. Chronic VLX treatment (20 mg/kg.day) for 4 weeks alleviated depression-like behavior in CUS rats in the OFT and FST. When the dose of VLX used in this study is converted to a human dose, it is $195 \mathrm{mg} / \mathrm{day}$ based on the body surface area normalization method (27). This converted dose is in the normal range of human dosages (the recommended maximum dosage of VLX is $225 \mathrm{mg} / \mathrm{day}$ orally) (28). The dose of VLX in this study was also reported effective in previous studies $(29,30)$. In addition, it was shown that chronic VLX treatment not only mitigated oxidative stress, but also prevented the decrease in TH and TPH and inhibited the overexpression of IDO and MAO-A, suggesting that its antidepressant effect may involve mitigating oxidative stress and augmenting monoamine neurotransmitter synthesis. 

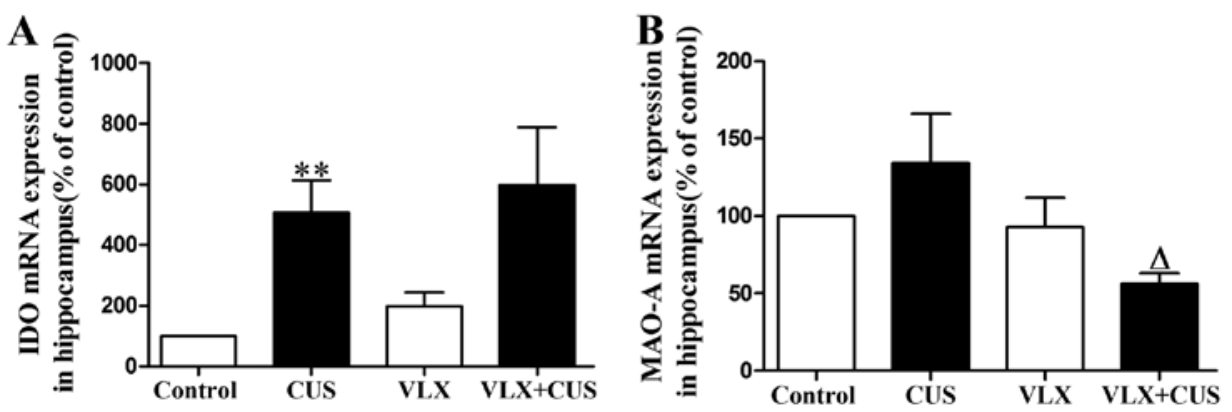

Figure 5. Effect of CUS on IDO and MAO-A mRNA expression in the rat hippocampus, and the effect of intervention with VLX. (A) IDO mRNA expression. (B) MAO-A mRNA expression. Mean $\pm \mathrm{SEM}, \mathrm{n}=5{ }^{* *} \mathrm{P}<0.01$ vs. control; ${ }^{\wedge} \mathrm{P}<0.05$ vs. CUS. CUS, chronic unpredictable stress; VLX, venlafaxine; IDO, indoleamine-2,3-dioxygenase; MAO-A, type A monoamine oxidase.
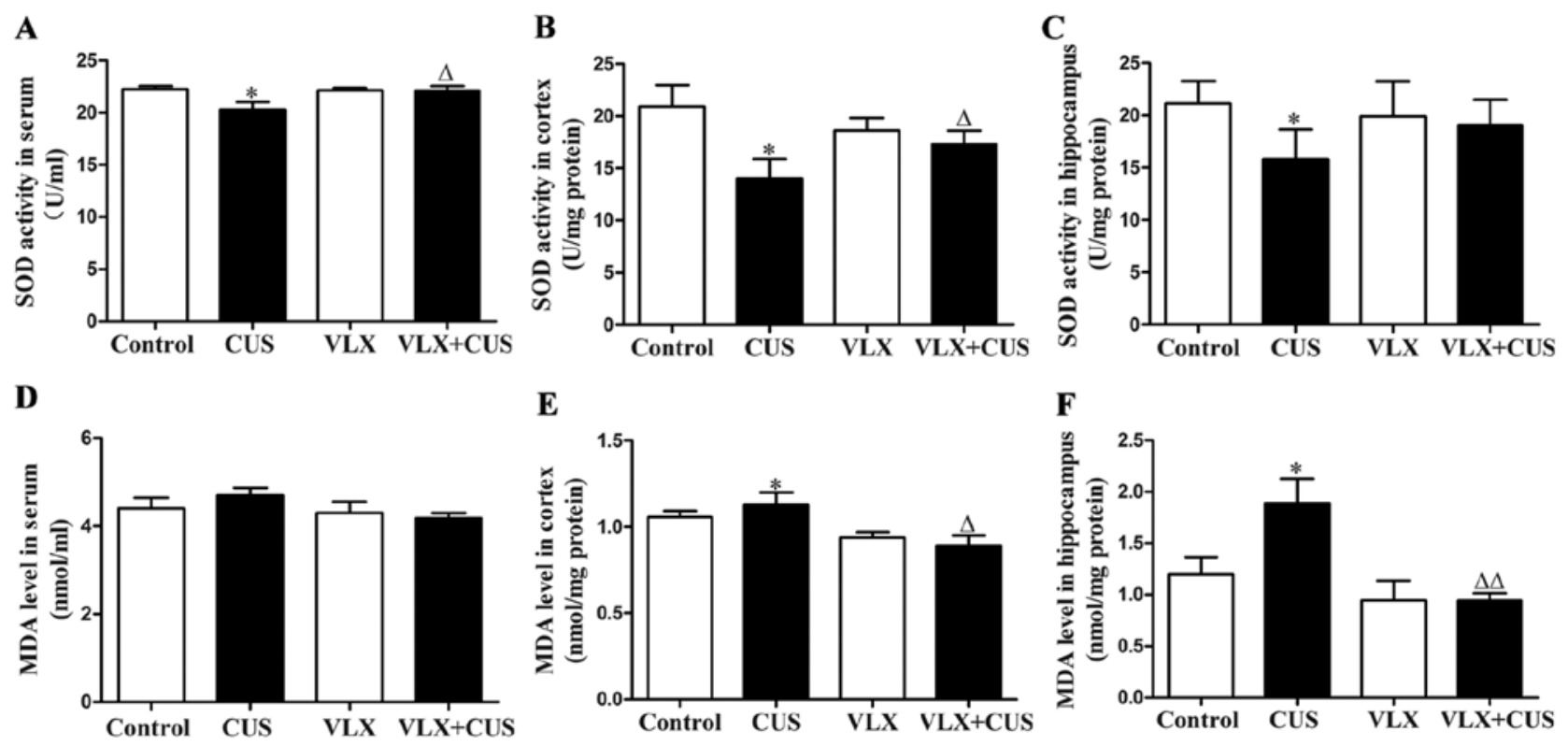

Figure 6. Effect of CUS on SOD activity and MDA content in the rat serum, cortex and hippocampus, and the effect of intervention with VLX. SOD activity in the (A) serum, (B) cortex and (C) hippocampus was assessed. The MDA content of the (D) serum, (E) cortex and (F) hippocampus was measured. Mean \pm SEM, $\mathrm{n}=10 .{ }^{*} \mathrm{P}<0.05$ vs. control; ${ }^{\Delta} \mathrm{P}<0.05,{ }^{\Delta \Delta} \mathrm{P}<0.01$ vs. CUS. CUS, chronic unpredictable stress; VLX, venlafaxine; SOD, superoxide dismutase; MDA, malondialdehyde.

The dysregulation of TH and TPH has been linked to the pathogenesis of depression, due to their roles in synthesis of monoaminergic transmitters. As previously reported, decreased TH protein and mRNA expression in the hippocampus may be observed in mice with depression caused by repeated injections of corticosterone (31). Following daily administration $(5 \mathrm{mg} / \mathrm{kg}$, i.p.) of fluoxetine for 2 weeks, the $\mathrm{TH}$ mRNA levels markedly increase in the rat locus coeruleus (32). In addition, evidence suggests that, when repeatedly separated from their pups, maternal rats become depressed, exhibiting lower expression of 5-HT and TPH in the dorsal raphe (33). Rats receiving fluoxetine treatment exhibit an increase in TPH expression in the dorsal raphe (7). Similarly, in the present study, the mRNA and protein TH and TPH expression in the cortex and hippocampus was generally decreased in the CUS rats, which was prevented by VLX treatment. These data showed that CUS might induce depressive symptoms by decreasing the synthesis of monoamine neurotransmitters. It is also possible that VLX exerted an antidepressant effect not only through the short-term reuptake inhibition of serotonin and noradrenalin, but also via long-term promotion of monoamine neurotransmitter synthesis. On the contrary, it has been reported that chronic restraint stress induces anxiety and depression-like behaviors accompanied by an increased TH mRNA expression in the nucleus accumbens, with fluoxetine treatment able to reverse this (34). Moreover, the protein expression of TPH was found to be increased by chronic social stress and normalized by citalopram (35). Interestingly, another study showed that chronic fluoxetine treatment raised TPH immunoreactivity in treated adolescent rats, while it reduced TPH immunoreactivity in treated adult rats in total dorsal raphe nuclei (36). Based on previous studies and the present results, these discrepancies in the expression of $\mathrm{TH}$ and TPH may be due to the variety of depression models, disparities in animal age, diversity of brain regions, and differences among the antidepressants used. However, further research is required to determine how SNRIs mediate the TH and TPH expression.

Tryptophan metabolism mediated by the kynurenine pathway may directly affect the synthesis of 5-HT. During this process, IDO catalyzes the conversion of tryptophan to kynurenine, usually via oxidative stress and cell-mediated 
immune activation (37). The upregulation of IDO is considered to be a biomarker of depressive disorder. A previous study reported that IDO was upregulated in the cortex following exposure to mild CUS, with markedly increased tumor necrosis factor (TNF)- $\alpha$ expression in the plasma and cerebral cortex (38). In a clinical study, it was shown that peripheral interferon (IFN)- $\alpha$ treatment led to IDO activation, ultimately causing depressive symptoms (39). It was shown that imipramine reduced IDO expression in both the hippocampus and raphe nuclei of rats exposed to chronic mild stress; however, these effects were not statistically significant (40). The present study showed that CUS led to a large increase in IDO expression in the hippocampus, with a generally increased MDA content and decreased SOD activity, indicating that CUS may inactivate 5-HT synthesis, partly through the IDO-induced kynurenine pathway of tryptophan metabolism. Interestingly, although VLX treatment clearly reversed the changes in oxidative stress parameters, it did not have an effect on IDO overexpression, suggesting that the antidepressant effect of VLX may involve the prevention of the harmful effects of oxidative stress, but not the correction of IDO overexpression. This result was similar to that of a previous study, which found that neither VLX alone, nor VLX in combination with agomelatine, had an effect on IDO activity in mice with chronic stress (41). This confirmed that the antidepressant effects of VLX were not mediated by its effect on the kynurenine pathway, at least in the present model.

Furthermore, an increasing body of evidence suggests that the monoamine metabolic enzyme MAO-A is a key regulator of depressive disorder $(42,43)$. 5-HT, an MAO-A substrate, has been known to be essential for neuronal plasticity and central to the pathogenesis of depression and age-related neurological diseases (44). MAO-A is not only present by heredity, but also by environmental factors, including hormonal factors and stress. An early study showed that salivary MAO-A activity was closely linked to stress (45). A previous study showed that the MAO-A expression was increased in the losing male mice following repeated experiences of social defeat, as compared with the winners and controls (46). It was also reported that MAO-A binding is elevated in postpartum or perimenopausal depression $(47,48)$. In fact, MAO-A activity can be inhibited by several types of antidepressants, including fluoxetine and VLX, suggesting that a decrease in MAO-A activity may be linked to the effects of the drugs on serotonergic, noradrenergic and dopaminergic neurotransmission (49). The present study showed that the MAO-A mRNA expression was generally elevated in the hippocampi of CUS rats, suggesting that chronic stress may cause depressive symptoms via the disruption of the metabolism of monoamine neurotransmitters. Long-term venlafaxine intervention clearly inhibited MAO-A overexpression, indicating that its antidepressant effect may involve weakening the metabolic abnormalities of monoamine neurotransmitters.

In conclusion, depression is usually the outcome of interactions between genetic and environmental factors, and its pathophysiology involves alterations of key enzymes responsible for monoamine neurotransmitter synthesis and metabolism. The therapeutic effect of VLX appears to upregulate the TH and TPH and decrease the MAO-A expression, indicating that strategies that facilitate monoamine neurotransmitter synthesis and correct metabolic disturbance may be beneficial for human depressive disorder. Moreover, VLX treatment mitigated oxidative stress, suggesting its importance in the protection of neurons from invasion, which is essential for neuroplasticity. However, VLX failed to have an effect on IDO overexpression, highlighting the need for more comprehensive and individualized treatments focusing on human depression. This article studied alterations in certain enzymes involved in the synthesis and metabolism of monoamine neurotransmitters. However, future research is required to explain the exact mechanism.

\section{Acknowledgements}

Not applicable.

\section{Funding}

The present study was partly supported by a grant from The National Natural Science Foundation of China (grant no. 31400881).

\section{Availability of data and materials}

The datasets used or analyzed during the present study are available from the corresponding author on reasonable request.

\section{Authors' contributions}

The work presented here was carried out by all authors in collaboration. DL and Q-XZ designed the research scheme. DL, X-YH, L-JW carried out the experiments. DL, H-JX, PS and X-PC were involved in data collection and analyzed the data. DL wrote the manuscript. Q-XZ revised the manuscript and took overall responsibility.

\section{Ethics approval and consent to participate}

All experimental procedures were approved by The Ethics Committee of Chongqing Medical University.

\section{Patient consent for publication}

Not applicable.

\section{Competing interests}

The authors declare that they have no competing interests.

\section{References}

1. Hamon $\mathrm{M}$ and Blier P: Monoamine neurocircuitry in depression and strategies for new treatments. Prog Neuropsychopharmacol Biol Psychiatry 45: 54-63, 2013.

2. Biegon A and Fieldust S: Reduced tyrosine hydroxylase immunoreactivity in locus coeruleus of suicide victims. Synapse 10: 79-82, 1992.

3. Dunčko R, Kiss A, Škultétyová I, Rusnák M and Jezová D: Corticotropin-releasing hormone mRNA levels in response to chronic mild stress rise in male but not in female rats while tyrosine hydroxylase mRNA levels decrease in both sexes. Psychoneuroendocrinology 26: 77-89, 2001.

4. Heydendael W and Jacobson L: Widespread hypothalamic-pituitary-adrenocortical axis-relevant and mood-relevant effects of chronic fluoxetine treatment on glucocorticoid receptor gene expression in mice. Eur J Neurosci 31: 892-902, 2010. 
5. Avgustinovich DF, Alekseenko OV, Bakshtanovskaia IV, Koriakina LA, Lipina TV, Tenditnik MV, Bondar' NP, Kovalenko IL and Kudriavtseva NN: Dynamic changes of brain serotonergic and dopaminergic activities during development of anxious depression: Experimental study. Usp Fiziol Nauk 35: 19-40, 2004 (In Russian).

6. Savelieva KV,Zhao S, Pogorelov VM, Rajan I, Yang Q, Cullinan E and Lanthorn TH: Genetic disruption of both tryptophan hydroxylase genes dramatically reduces serotonin and affects behavior in models sensitive to antidepressants. PLoS One 3: e3301, 2008.

7. Yang FZ, Wu Y, Zhang WG, Cai YY and Shi SX: Estradiol or fluoxetine alters depressive behavior and tryptophan hydroxylase in rat raphe. Neuroreport 21: 309-312, 2010.

8. Shishkina GT, Kalinina TS and Dygalo NN: Up-regulation of tryptophan hydroxylase- 2 mRNA in the rat brain by chronic fluoxetine treatment correlates with its antidepressant effect. Neuroscience 150: 404-412, 2007.

9. Kim SW, Park SY and Hwang O: Up-regulation of tryptophan hydroxylase expression and serotonin synthesis by sertraline. Mol Pharmacol 61: 778-785, 2002.

10. Qin Y, Wang N, Zhang X, Han X, Zhai X and Lu Y: IDO and TDO as a potential therapeutic target in different types of depression. Metab Brain Dis 33: 1787-1800, 2018.

11. Christmas DM, Potokar J and Davies SJ: A biological pathway linking inflammation and depression: Activation of indoleamine 2,3-dioxygenase. Neuropsychiatr Dis Treat 7: 431-439, 2011.

12. Samelson-Jones BJ and Yeh SR: Interactions between nitric oxide and indoleamine 2,3-dioxygenase. Biochemistry 45: 8527-8538, 2006.

13. Myint AM and Kim YK: Cytokine-serotonin interaction through IDO: A neurodegeneration hypothesis of depression. Med Hypotheses 61: 519-525, 2003.

14. Dantzer R, O'Connor JC, Freund GG, Johnson RW and Kelley KW: From inflammation to sickness and depression: When the immune system subjugates the brain. Nat Rev Neurosci 9: 46-56, 2008.

15. Dobos N, deVries EF, Kema IP, Patas K, Prins M, Nijholt IM, Dierckx RA, Korf J, den Boer JA, Luiten PG and Eisel UL: The role of indoleamine 2,3- dioxygenase in a mouse model of neuroinflammation-induced depression. J Alzheimers Dis 28 905-915, 2012.

16. Stefanescu $\mathrm{C}$ and Ciobica A: The relevance of oxidative stress status in first episode and recurrent depression. J Affect disord 143: 34-38, 2012.

17. Naoi M, Maruyama W and Shamoto-Nagai M: Type A monoamine oxidase and serotonin are coordinately involved in depressive disorders: From neurotransmitter imbalance to impaired neurogenesis. J Neural Transmission (Vienna) 125 53-66, 2018

18. Buckholtz JW and Meyer-Lindenberg A: MAOA and the neurogenetic architecture of human aggression. Trends Neurosci 31: $120-129,2008$

19. Meyer JH, Ginovart N, Boovariwala A, Sagrati S, Hussey D Garcia A, Young T, Praschak-Rieder N, Wilson AA and Houle S: Elevated monoamine oxidase a levels in the brain: An explanation for the monoamine imbalance of major depression. Arch Gen Psychiatry 63: 1209-1216, 2006.

20. Grunewald M, Johnson S, Lu D, Wang Z, Lomberk G, Albert PR, Stockmeier CA, Meyer JH, Urrutia R, Miczek KA, et al Mechanistic role for a novel glucocorticoid-KLF11 (TIEG2) protein pathway in stress-induced monoamine oxidase A expression. J Biol Chem 287: 24195-24206, 2012.

21. Bonnet U: Moclobemide: Therapeutic use and clinical studies. CNS Drug Rev 9: 97-140, 2010.

22. Elhwuegi AS: Central monoamines and their role in major depression. Prog Neuropsychopharmacol Biol Psychiatry 28 435-451, 2004

23. Ye Y, Wang G, Wang $\mathrm{H}$ and Wang X: Brain-derived neurotrophic factor (BDNF) infusion restored astrocytic plasticity in the hippocampus of a rat model of depression. Neurosci Lett 503: 15-19, 2011.

24. Grundmann O, Lv Y, Kelber O and Butterweck V: Mechanism of St. John's wort extract (STW3-VI)during chronic restraint stress is mediated by the interrelationship of the immune, oxidative defense, and neuroendocrine system. Neuropharmacology 58 767-773, 2010

25. Makino M, Kitano Y, Komiyama $C$ and Takasuna K: Human interferon-alpha increases immobility in the forced swimming test in rats. Psychopharmacology (Berl) 148: 106-110, 2000.
26. Livak KJ and Schmittgen TD: Analysis of relative gene expression data using real-time quantitative PCR and the 2(-Delta Delta C(T)) method. Methods 25: 402-408, 2001.

27. Reagan-Shaw S, Nihal M and Ahmad N: Dose translation from animal to human studies revisited. FASEB J 22: 659-661, 2008.

28. Watanabe Y, Asami Y, Hirano Y, Kuribayashi K, Itamura R and Imaeda T: Factors impacting the efficacy of venlafaxine extended release $75-225 \mathrm{mg} /$ day in patients with major depressive disorder: Exploratory post hoc subgroup analyses of a randomized, double-blind, placebo-controlled study in Japan. Neuropsychiatr Dis Treat 14: 1261-1272, 2018.

29. Szkutnik-Fiedler D, Kus K, Balcerkiewicz M, Grześkowiak E, Nowakowska E, Burda K, Ratajczak P and Sadowski C: Concomitant use of tramadol and venlafaxine-evaluation of antidepressant-like activity and other behavioral effects in rats. Pharmacol Rep 64: 1350-1358, 2012.

30. Yilmaz N, Demirdas A, Yilmaz M, Sutcu R, Kirbas A, Cure MC and Eren I: Effects of venlafaxine and escitalopram treatments on NMDA receptors in the rat depression model. J Membr Biol 242: 145-151, 2011.

31. Zhao Y, Ma R, Shen J, Su H, Xing D and Du L: A mouse model of depression induced by repeated corticosterone injections. Eur J Pharmacol 581: 113-120, 2008.

32. Brady LS, Gold PW, Herkenham M, Lynn AB, Whitfield HJ Jr: The antidepressants fluoxetine, idazoxan and phenelzine alter corticotropin-releasing hormone and tyrosine hydroxylase mRNA levels in rat brain: Therapeutic implications. Brain Res 572: 117-125, 1992

33. Sung YH, Shin MS, Cho S, Baik HH, Jin BK, Chang HK, Lee EK and Kim CJ: Depression-like state in maternal rats induced by repeated separation of pups is accompanied by a decrease of cell proliferation and an increase of apoptosis in the hippocampus. Neurosci Lett 470: 86-90, 2010.

34. Zhao X, Seese RR, Yun K, Peng T and Wang Z: The role of galanin system in modulating depression, anxiety, and addiction-like behaviors after chronic restraint stress. Neuroscience 246: 82-93, 2013.

35. Abumaria N, Rygula R, Hiemke C, Fuchs E, HavemannReinecke U, Rüther E and Flügge G: Effect of chronic citalopram on serotonin-related and stress-regulated genes in the dorsal raphe nucleus of the rat. Eur Neuropsychopharmacol 17: 417-429, 2007.

36. Klomp A, Václavů L, Meerhoff GF, Reneman L and Lucassen PJ: Effects of chronic fluoxetine treatment on neurogenesis and tryptophan hydroxylase expression in adolescent and adult rats. PLoS One 9: e97603, 2014

37. Maes M, Leonard BE, Myint AM, Kubera M and Verkerk R: The new '5-HT' hypothesis of depression: Cell-mediated immune activation induces indoleamine 2,3-dioxygenase, which leads to lower plasma tryptophan and an increased synthesis of detrimental tryptophan catabolites (TRYCATs), both of which contribute to the onset of depression. Prog Neuropsychopharmacol Biol Psychiatry 35: 702-721, 2011.

38. Liu YN, Peng YL, Liu L, Wu TY, Zhang Y, Lian YJ, Yang YY, Kelley KW, Jiang CL and Wang YX: TNF $\alpha$ mediates stress-induced depression by upregulating indoleamine 2 , 3 -dioxygenase in a mouse model of unpredictable chronic mild stress. Eur Cytokine Netw 26: 15-25, 2015.

39. Raison CL, Dantzer R, Kelley KW, Lawson MA, Woolwine BJ, Vogt G, Spivey JR, Saito K and Miller AH: CSF concentrations of brain tryptophan and kynurenines during immune stimulation with IFN-alpha: Relationship to CNS immune responses and depression. Mol Psychiatry 15: 393-403, 2010.

40. Mohamed BM, Aboul-Fotouh S, Ibrahim EA, Shehata H, Mansour AA, Yassin NA, El-Eraky W and Abdel-Tawab AM: Effects of pentoxifylline, 7-nitroindazole, and imipramine on tumor necrosis factor- $\alpha$ and indoleamine 2,3-dioxygenase enzyme activity in the hippocampus and frontal cortex of chronic mild-stress-exposed rats. Neuropsychiatr Dis Treat 9: 697-708, 2013.

41. Thomas J, Khanam R and Vohora D: Augmentation of antidepressant effects of venlafaxine by agomelatine in mice are independent of kynurenine pathway. Neurochem Int 99: 103-109, 2016.

42. SchulzeTG,MüllerDJ,Krauss H,ScherkH,OhlraunS,Syagailo YV, Windemuth C, Neidt H, Grässle M, Papassotiropoulos A, et al: Association between a functional polymorphism in the monoamine oxidase A gene promoter and major depressive disorder. Am J Med Genet 96: 801-803, 2000. 
43. Duncan J, Johnson S and Ou XM: Monoamine oxidases in major depressive disorder and alcoholism. Drug Discov Ther 6: 112-122, 2012.

44. Mattson MP, Maudsley S and Martin B: BDNF and 5-HT: A dynamic duo in age-related neuronal plasticity and neurodegenerative disorders. Trends Neurosci 27: 588-594, 2004.

45. Doyle A, Hucklebridge F, Evans P and Clow A: Salivary monoamine oxidase A and B inhibitory activities correlate with stress. Life Sci 59: 1357-1362, 1996.

46. Filipenko ML, Beilina AG, Alekseyenko OV, Dolgov VV and Kudryavtseva NN: Repeated experience of social defeats increases serotonin transporter and monoamine oxidase A mRNA levels in raphe nuclei of male mice. Neurosci Lett 321: 25-28, 2002.
47. Sacher J, Wilson AA, Houle S, Rusjan P,Hassan S, Bloomfield PM, Stewart DE and Meyer JH: Elevated brain monoamine oxidase A binding in the early postpartum period. Arch Gen Psychiatry 67: 468-474, 2010.

48. Rekkas PV, Wilson AA, Lee VW, Yogalingam P, Sacher J, Rusjan P, Houle S, Stewart DE, Kolla NJ, Kish S, et al: Greater monoamine oxidase a binding in perimenopausal age as measured with carbon 11-labeled harmine positron emission tomography. JAMA Psychiatry 71: 873-879, 2014.

49. Fisar Z, Hroudová J and Raboch J: Inhibition of monoamine oxidase activity by antidepressants and mood stabilizers. Neuro Endocrinol Lett 31: 645-656, 2010. 